\title{
SIMULATION OF THE THERMAL HISTORY DEPENDENCE OF PRIMARY SPACING DURING DIRECTIONAL SOLIDIFICATION
}

\author{
Hongbiao Dong ${ }^{1}$, Wei Wang ${ }^{2}$, Peter D. Lee ${ }^{1}$ \\ ${ }^{1}$ Imperial College London, Prince Consort Road, SW7 2BP, U.K. \\ ${ }^{2}$ Alcoa Technical Center, 100 Technical Drive, Alcoa Center, PA 15069, USA
}

Keywords: Directional solidification, Cellular automaton, History dependence, Primary spacing, Solidification modeling

\begin{abstract}
Thermal history dependent primary dendrite spacing during directional solidification was investigated using a combined cellular automaton-finite difference model. This model simulates the key phenomena occurring during dendritic growth, including overgrowth and branching. The dependence of primary spacing upon the thermal history during columnar dendritic growth was studied for a range of thermal gradient from 1 to $18 \mathrm{~K} \mathrm{~mm}^{-1}$ and constant pulling velocity. Changes in primary spacing through both branching and overgrowth mechanisms were quantified. It was found that a range of stable spacings were possible for any given growth condition, with the maximum spacing about 3 times the minimum spacing. Further, for a monotonic increase in thermal gradient the spacing always stayed at the upper bound, whilst for a monotonic decrease the lower bound was followed. It was also found that the incubation period for dendrite branching at the upper limit was longer than that for overgrowth. The influence of having a range of spacings upon the propensity for equiaxed grains to nucleate ahead of the columnar dendrites was also investigated, revealing for the same thermal conditions, fine primary spacings are less prone to freckle formation.
\end{abstract}

\section{Introduction}

In castings there are many characteristic microstructural length scales such as dendrite tip radius and primary and secondary dendritic spacing. For single crystal applications where the structure is columnar dendrites, the primary spacing $\left(\lambda_{1}\right)$ characterizes the maximum length scale for the segregation of alloying elements, hence affecting the subsequent heat treatment process and the thermal mechanical properties of the alloys [1, 2]. A fine spacing is sought to minimize heat treatment time and provide a concomitant increase in mechanical properties. This is of particular importance for the second and third generation single crystal superalloys, which contain refractory elements with low diffusivities and hence long solutionizing time [3].

Over the past thirty years, considerable progress has been made in determining the influence of processing parameters upon the primary dendrite spacing through theoretical and experimental studies [4-7]. Early studies characterized the spacing as a unique value for a given condition, but latter studies have illustrated that a range of spacings is possible for any given set of growth conditions. Experimental observations suggested $[5,6]$ that the spacing range is governed by the overgrowth at the minimum and the branching at the maximum. Further, these studies indicated that the primary spacing is not only dependent on the current growth conditions, but also on the thermal history. Stability analysis of the solid/liquid $(\mathrm{S} / \mathrm{L})$ interface by perturbing the solidification front $[8,9]$ also indicated that a given spacing is stable over a range of velocities. Using this type of analysis the lower bound in an allowable velocity range can be calculated, but not the upper bound. Calculations from a numerical model developed by Hunt et al. [10] suggested a solute interaction limit to determine the lower band of spacing. Branching was not included in their model, and therefore the upper limit was estimated to be twice the lower limit. To gain a better understanding of the primary spacing, models including both overgrowth and branching are required.

Several numerical techniques are available which might be used to study these phenomena. Phase field (PF) methods can simulate most of the dendritic features observed experimentally, including branching. However, a very fine grid size $(<1 \mu \mathrm{m})$ is required to capture the solid-liquid boundary layer, limiting the size of domains which can be simulated (e.g. $<1 \mathrm{~mm}^{2}$ in 2D) [11]. Cellular automaton methods have been coupled with finite element (CAFE) models to simulate grain structures during solidification [12]; however, these techniques do not capture the same level of detail as PF methods. CAFE models do not incorporate details of dendritic growth, but they are very useful for simulating grain structures on orders of magnitude larger simulation domains than is possible using PF. The CAFE model is based on the concept of marginal stability to uniquely define the dendrite tip radius, allowing the analytical solution of Kurz, Giovanola and Trivedi (KGT) to be applied [13]. Using the KGT model to relate thermal undercooling to the restriction in grain growth requires only the thermal field to be solved, not the solute field. CAFE models do not simulate solute interaction; therefore variations in primary dendrite spacing, branching and overgrowth cannot be modeled.

Another modeling technique available is based on combining the cellular automaton (CA) method for tracking the solid-liquid front location with a finite difference (FD) solution of solute diffusion, termed a CA-FD model. This model has been applied by the current authors and co-workers to simulate dendritic growth in a range of alloys [14-19]. The CA-FD model does not use an analytical solution to determine the velocity of the solid-liquid $(\mathrm{S} / \mathrm{L})$ interface and radius of the dendritic tip, but instead solves the solute conservation equation subjected to equilibrium conditions at the $\mathrm{S} / \mathrm{L}$ interface. The model simulates the solutal interaction within the developing dendritic network, predicting when overgrowth or branching will occur. The model has been previously applied to investigate the effect of changing the pulling velocity on directionally solidified dendritic structures in Ni-based superalloys [19]. A range of possible spacings was predicted, which agrees well with analytic calculations and experimental measurements (see Fig. 1). 


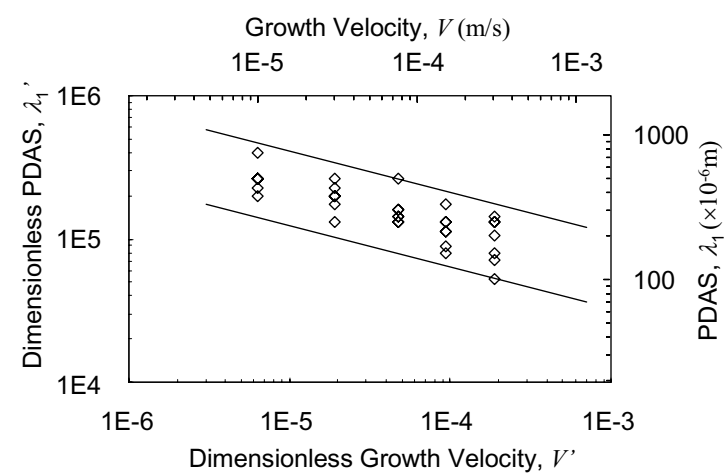

Fig. 1 Predicted primary dendrite spacing as a function of pulling velocity. The lower and upper bounds in spacing are plotted as solid lines. (After [19].)

In this paper, the CA-FD model is used to investigate thermal history dependence during directional solidification. The model theory is outlined first, followed by a comparison of predicted spacings with analytical calculations under constant conditions for model validation. The thermal history dependence is then investigated by determining the influence of ramping the thermal gradient upon the primary spacing. Finally, the effect of the primary spacing on stray grain and equiaxed freckle chain formation is explored.

\section{Model Theory}

The model simulates the evolution of dendritic structures in a binary system in three dimensions by solving for solute diffusion and surface tension effects upon the growth velocity of the $\mathrm{S} / \mathrm{L}$ interface. The influence of crystallographic anisotropy is incorporated by an adaptation of the decentered square growth algorithm proposed by Gandin and Rappaz [20]. A detailed description of the model formulation has been presented previously [19]; therefore, only a brief description of the model together with the key assumptions and limitations are presented below.

\section{Model Description}

Although the CA-FD is a 3D model, simulations in this study were performed on a 2D domain. A schematic representation of the simulation domain and thermal profile is shown in Fig. 2. A zero flux boundary condition was applied to the left and right sides of the domain. A temporally varying thermal field with a vertical spatial gradient was imposed upon the domain to simulate the thermal conditions during directional solidification. The domain was discretized into a regular grid of cells, each $5 \mu \mathrm{m}$ square. Both the CA and FD components of the model use the same cell size and time step.

Each cell has three possible states: liquid, solid and 'growing' (i.e. a mixture of liquid and solid). As an initial condition, a few of the cells at the bottom of the domain had their state set to 'growing', forming 'seeds'; all the other cells were initially set to 'liquid'. Within each 'growing' cell, solute partitioning occurs, and excess solute is rejected from the $\mathrm{S} / \mathrm{L}$ interface, increasing the concentration in liquid ahead of the interface. This buildup is reduced by diffusion, transporting solute to neighboring cells.

Solute diffusion is calculated using an explicit finite difference method, and the change of solid fraction $\left(f_{S}\right)$ in a 'growing' cell is

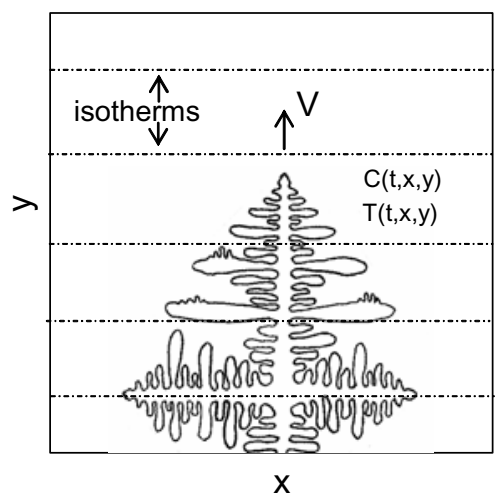

Fig. 2 Schematic representation of simulation domain and imposed thermal profile.

determined by the variation of solute concentration within the cell and the equilibrium concentration at the $\mathrm{S} / \mathrm{L}$ interface. In the model, equilibrium concentration is derived from the phase diagram, assuming that equilibrium occurs at the $\mathrm{S} / \mathrm{L}$ interface. The calculated fraction solid change is used to correlate the growth rate in a growing cell according to the decentered square algorithm [19]. This method allows the preferred growth directions of cubic metals to be maintained during the simulations, independent of the grain's orientation to the square solution grid.

A moving frame of reference technique was also used in the simulations. When the temperature at the bottom decreases to a predetermined value, the bottom layer of the cells is removed from the simulation domain, and all the other layers are then shifted down by one cell and a new layer with the initial concentration is added to the top of the domain. This technique allows a much smaller solution domain to be used, reducing the computational expense.

An important feature of the present model is that there is no predetermined shape for the dendrite tip or the dendrite itself, and the variations in shape are determined by the solutal interaction, fraction solid evolution and the growth algorithm, including the time dependency.

\section{Assumptions and Limitations of the Model}

In the CA-FD model used, only the solute balance, and not the heat balance, is equilibrated at the $\mathrm{S} / \mathrm{L}$ interface. This assumption is not valid for very dilute compositions or pure metals where growth is controlled by the diffusion of heat from the interface, rather than solute. For growth controlled by the solute diffusion, the error in thermal profile due to latent heat evolution is not significant, which is the case for most alloys in industrial directional solidification or single crystal growth furnaces.

The kinetic undercooling effect on the equilibrium temperature and composition is not included, so the model is only valid for growth at low Péclet numbers ( $P e=D \times V / \alpha<1)$, where $D$ is the characteristic length, $V$ is the growth velocity and $\alpha$ is thermal diffusivity. Therefore the model is not applicable to rapid solidification processes.

In addition, the equilibrium data is obtained from a linearised phase diagram for a binary system, and the partition coefficient, diffusion coefficients and slope of the liquidus line were assumed to be constant. 
Table 1 Material properties and model parameters used in the simulations.

\begin{tabular}{ccc}
\hline Property & Variable & Value \\
\hline Liquidus temperature & $T_{L I Q}$ & $1609 \mathrm{~K}$ \\
Liquidus slope & $m$ & $-8.9 \mathrm{~K}(\mathrm{wt} \%)^{-1}$ \\
Partition coefficient & $k$ & 0.48 \\
Diffusion coefficient in liquid & $D_{L}$ & $3.0 \times 10^{-9} \mathrm{~m}^{2} \mathrm{~s}^{-1}$ \\
Diffusion coefficient in solid & $D_{S}$ & $3.0 \times 10^{-12} \mathrm{~m}^{2} \mathrm{~s}^{-1}$ \\
Gibbs Thomson coefficient & $\Gamma$ & $1.0 \times 10^{-7} \mathrm{~K} \cdot \mathrm{m}$ \\
Initial concentration & $C_{0}$ & $4.85 \mathrm{wt} \%$ \\
Cell size & $\Delta x$ & $5 \mu \mathrm{m}$ \\
Time step & $\Delta t$ & $1.0 \times 10^{-3} \mathrm{~s}$ \\
Pulling velocity & $V$ & $150 \mu \mathrm{m} \mathrm{s}^{-1}$ \\
Thermal gradient & $G$ & $1 \sim 18 \mathrm{~K} \mathrm{~mm}^{-1}$ \\
\hline
\end{tabular}

\section{Validation of the Model for Primary Spacing}

Numerical models always have some level of error resulting from truncation effects, artificial solute trapping and interface spreading, all leading to a cell size dependency [21]. To illustrate the capabilities of the present model, the effect of cell size on the solutal interaction and spacing were investigated first, followed by numerical stability analysis of the calculation. Then a detailed comparison of predicted primary spacing with those obtained from analytical solutions and experimental observations is presented.

\section{$\underline{\text { Cell Size Dependency }}$}

In the CA-FD model, the computational time increases dramatically when the cell size $(\Delta x)$ decreases for a given domain size; it is inversely proportional to $(\Delta x)^{4}$ for $2 \mathrm{D}$ simulations. Thus the largest cell size, which still captures the required dendritic features, is preferred, to reduce computational times.

To investigate the cell size dependence, a set of numerical simulations was performed with cell sizes ranging from $1 \mu \mathrm{m}$ to $20 \mu \mathrm{m}$. A binary approximation to a nickel-based superalloy was used; the material properties are given in Table 1. Simulations were run for the conditions of a pulling velocity, $V$, of $150 \mu \mathrm{m} \mathrm{s}^{-1}$ and a thermal gradient, $G$, of $12 \mathrm{~K} \mathrm{~mm}^{-1}$. The domain size was $1.5 \times 1.5 \mathrm{~mm}^{2}$ except for the case with $1 \mu \mathrm{m}$ cells, where a domain of $0.75 \times 0.75 \mathrm{~mm}^{2}$ was used. Calculations started with the same initial conditions, including seed locations and spacing $(300 \mu \mathrm{m})$.

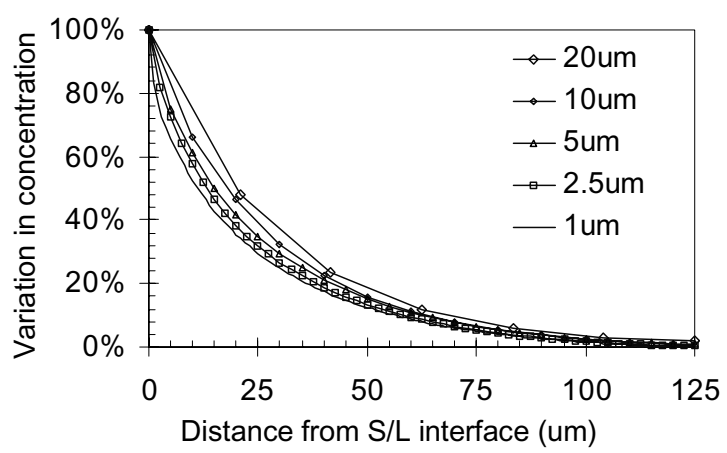

Fig. 3 The variation in solute concentration at the dendrite tip in a direction parallel with the isotherms.

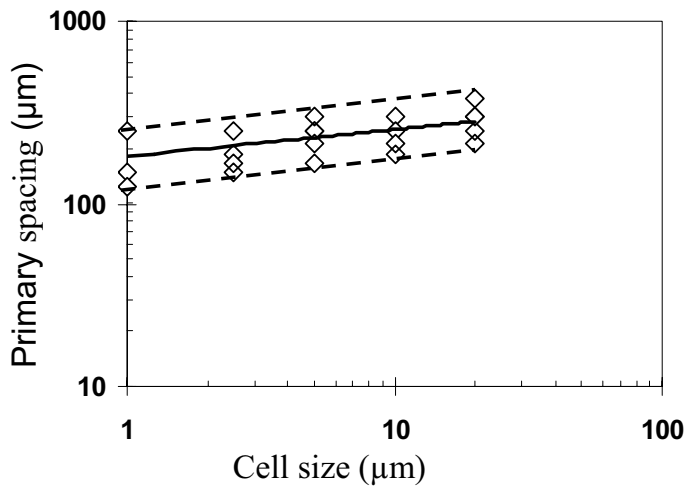

Fig. 4 Variation of stable primary spacings against cell size, the solid line is the average value.

Fig. 3 shows the concentration variation, defined as $\left(C_{L}-C_{\infty}\right) /\left(C_{L}^{*}-C_{\infty}\right)$, at the primary dendrite tips along the direction parallel to the isotherms, where $C_{L}, C_{L}^{*}, C_{\infty}$ are solute concentration in liquid, at the interface and in the bulk, respectively. The model monotonically converges to the solute curve defined by the finest spacing, with the largest deviation being on the coarsest grid due to discretization error and lack of definition of the tip curvature [21].

Using the decentered square algorithm also introduces a dependency of the tip radius upon cell size, which in turn affects the primary spacing. This is due to the algorithm forcing the crystal anisotropy to be of a sharpness of a single cell at the point of the square (or octahedron in 3D). Therefore, simulations were carried out to determine the range of stable primary spacing as a function of cell size (see Fig. 4). All the points are located within a narrow band demarcated by two dashed lines parallel with the solid line. With reducing cell size a small dependency of spacing is observed, as discussed in detail in [22].

Due to the minor dependency of spacing on cell size and to minimize the error in solute field, a cell size of $5 \mu \mathrm{m}$ was selected as a reasonable trade-off between speed and accuracy, with the average run taking 2 hours on a Pentium PC.

\section{Effect of Time Step}

An explicit numerical solution technique was used for solute diffusion, hence the time step, $\Delta t$, has to be small enough to

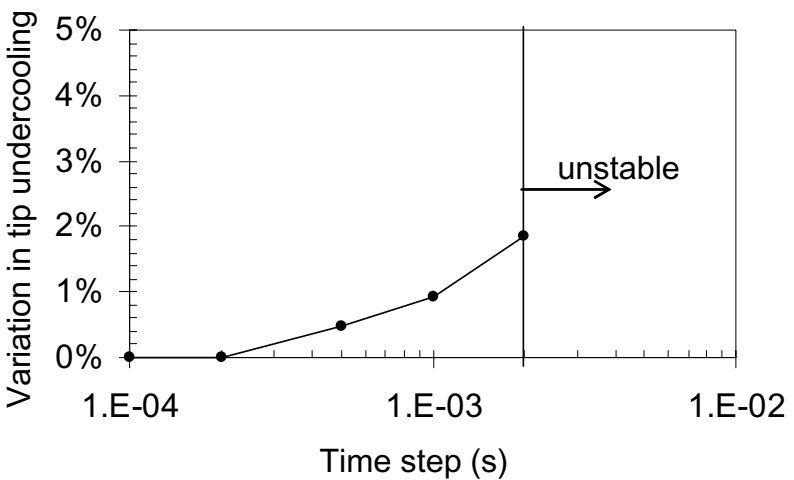

Fig. 5 Tip undercooling as a function of time step. 
satisfy the Courant stability requirement, or $\Delta t \leq 1 / 4\left(\Delta x^{2} / D_{L}\right)$ for $2 \mathrm{D}$ simulations. For a cell size of $5 \mu \mathrm{m}$, this sets the upper bound on $\Delta t$ to be $2.1 \times 10^{-3} \mathrm{~s}$. A series of simulations were carried out using the conditions listed in the previous section for a cell size of $5 \mu \mathrm{m}$ but varying the time step from $1 \times 10^{-4}$ to $3 \times 10^{-3} \mathrm{~s}$. As expected, the computation was unstable when $\Delta t=3 \times 10^{-3} \mathrm{~s}$. All the other simulations were stable, producing qualitatively identical results but with a monotonically reducing error in the predicted tip undercooling (see Fig 5). On decreasing $\Delta t$ by a factor of 10 from $1 \times 10^{-3}$ to $1 \times 10^{-4}$, the tip undercooling is affected by only $1 \%$, therefore a time step of $1 \times 10^{-3} \mathrm{~s}$ was used for computational efficiency.

\section{Comparison with Analytic Models and Experiments}

To further validate the models, the predicted primary dendrite spacings under constant conditions were compared with those obtained from prior analytical and numerical solutions. The comparison was made for a constant pulling velocity of $150 \mu \mathrm{m} \mathrm{s}^{-1}$ and the thermal gradient was varied from 1 to $18 \mathrm{~K}$ $\mathrm{mm}^{-1}$. The predicted dendritic morphologies are shown in Fig. 6 starting with 2 seeds for four gradients in this range. The models were run for $40 \mathrm{~s}$ of simulation time (sufficient to stabilize primary dendrite spacing). The strong dependence of primary spacings upon thermal gradient can be seen, with $\lambda_{1}$ decreasing from 1500 to $176 \mu \mathrm{m}$ as $G$ is increased from 1 to $18 \mathrm{~K} / \mathrm{mm}$.

Previous experiments and simulations suggested that there exists a wide range of stable primary dendrite spacings under any given growth condition, and that the initial nucleation conditions can have an effect on the final spacing [19]. For this reason, the model was rerun for each thermal gradient and with different numbers of initial seeds $(2,4,8,12,20$ and 30). To compare with analytical calculations, the distribution of dimensionless average primary dendrite spacing was considered, using the non-dimensional parameters suggested by Hunt and $\mathrm{Lu}$ [10]. The average dimensionless spacing, defined as $\left(\lambda^{\prime}=\lambda m C_{0} / \Gamma\right)$, plotted against dimensionless thermal gradient $\left(G^{\prime}=G \Gamma /\left(m C_{0}\right)^{2}\right)$ for various

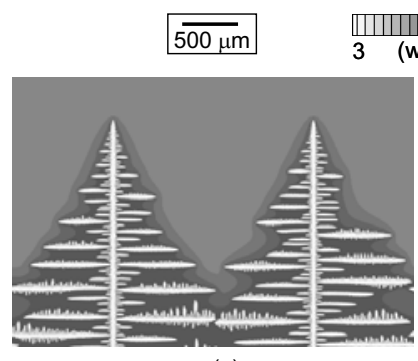

(a)

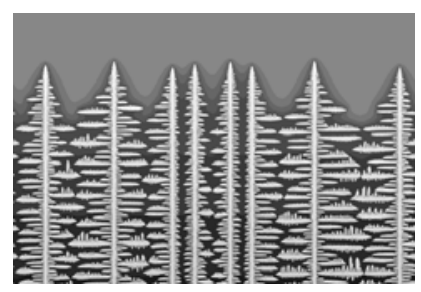

(c)

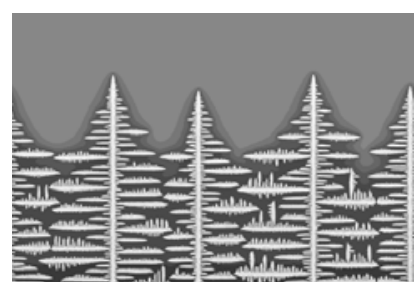

(b)

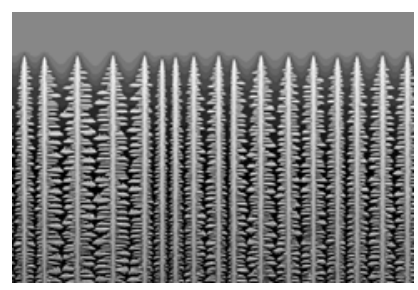

(d)
Fig. 6 Predicted dendritic structures (from 2 seeds) for thermal gradients of: (a) 1 , (b) 3 , (c) 6 and (d) $18 \mathrm{~K} \mathrm{~mm}^{-1}$. initial nucleation conditions at different solidification rates, is shown in Fig. 7. (Note: the parameters used in non-dimensionalizing are: $m$, the liquidus slope; $\Gamma$, the Gibbs Thomson Coefficient; and $C_{0}$, the initial solute concentration.) For each gradient a range of spacings is predicted by the model depending upon the initial seed spacing, all lying within the narrow band demarcated by the two parallel lines:

and

$$
\lambda_{1 \min }^{\prime}=39.2\left(G^{\prime}\right)^{-0.51}
$$

$$
\lambda_{1 \max }^{\prime}=128\left(G^{\prime}\right)^{-0.51} .
$$

These two power-law relationships give the lower and upper limits of the allowable primary dendrite spacing, with the upper approximately three times the lower. The value of the exponent is almost equal to 0.5 . This is the value predicted by prior analytic models [23], and slightly higher than the range of values predicted by the numerical model of Hunt and $\mathrm{Lu}[10]$. The predicted stable spacing agrees well with prior analytical and numerical models, as well as experiment. The benefit of the current model is its ability to produce realistic dendritic structures and hence the variation in spacing, which the other models cannot.

\section{Primary Spacing History Dependence}

In this section, columnar dendritic growth was simulated for conditions of a constant pulling rate but a ramping thermal gradient, increasing from 1 to $18 \mathrm{~K} \mathrm{~mm}^{-1}$ in 30 steps (a 1.1 times increase between each step). The thermal gradient was then ramped down by over the same range in 30 steps, by a factor of 0.91 . The resulting input ramp and resulting primary spacing is shown in Fig 8. Each step was maintained for $10 \mathrm{~s}$, except for the last few steps, which were $20 \mathrm{~s}$ long. The history dependence of primary spacing is illustrated by the hysteresis in primary spacing in Fig 8b- the upper bound (eqn. (2)) is hugged whilst increasing the gradient, and the lower bound (eqn. (1)) is hugged whilst monotonically decreasing the gradient. The dendritic structures corresponding to points ' $a$ ' to ' $h$ ' in Fig. 8(b) are shown in Fig. 9(a) to (h), respectively.

The simulations start with a coarse dendrite spacing, point 'a', where $\lambda_{1}=1500 \mu \mathrm{m}\left(G=1 \mathrm{~K} \mathrm{~mm}^{-1}\right)$. As shown in Fig. 9(a), two columnar dendrites grow from the bottom, with secondary and tertiary dendrites forming on the primary branches. As $G$

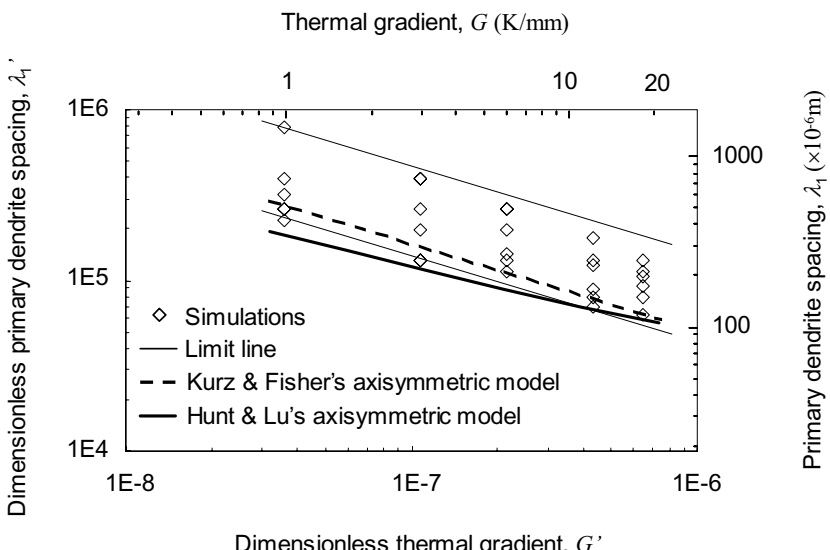

Fig. 7 Predicted primary spacing as a function of thermal gradient, together with the analytical solution of Kurz and Fisher[23] and the numerical model of Hunt and Lu[11]. 

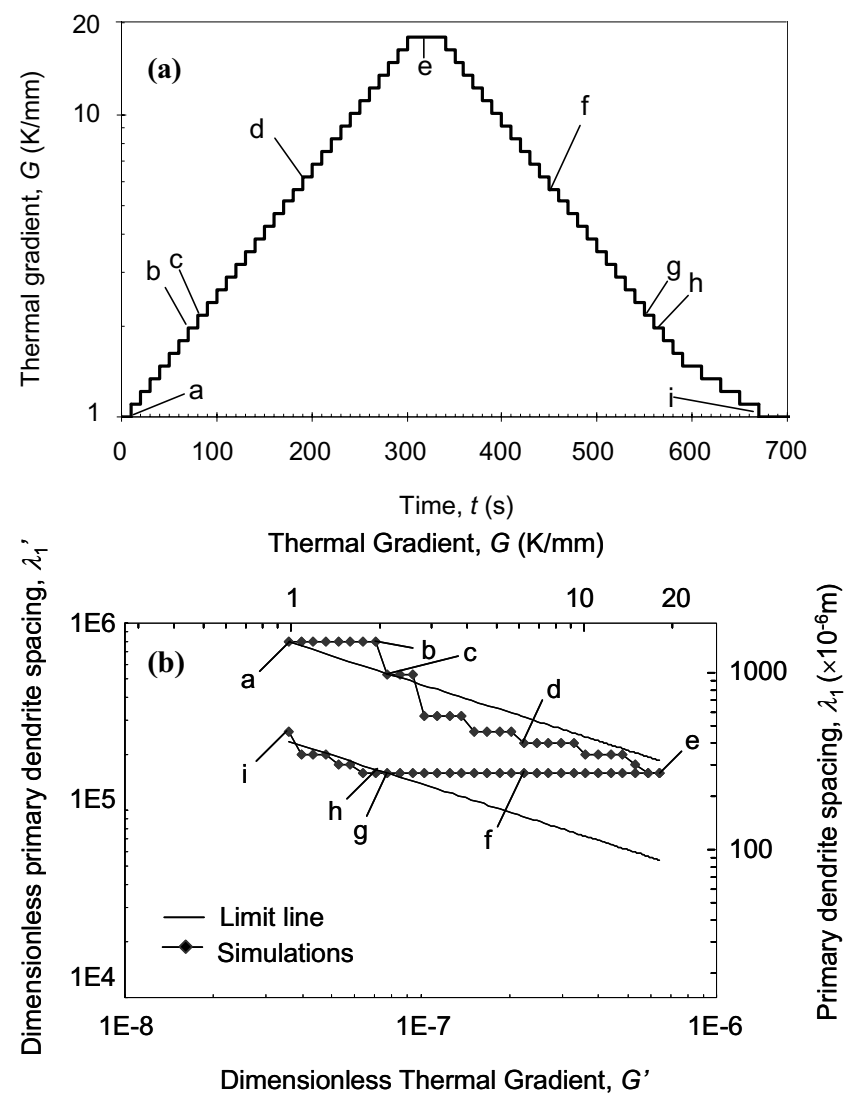

Fig. 8 (a) Thermal gradient input into the simulation. (b) Resulting average primary dendrite spacing. (Note, the dendritic structures corresponding to points (a) to (i) are shown in Fig. 9.)

increases, the tertiary arms become elongated in the pulling direction, but are blocked from forming new, stable, primaries by secondary arms ahead of them (see Fig. 9(b)). The primary spacing remains constant despite the increase in $G$ until a sufficiently great change is reached, or $G=2.16 \mathrm{~K} \mathrm{~mm}^{-1}$. During this step, one of the tertiary dendrites successfully penetrates the blockage of secondary arms (see Fig. 9(c)) and $\lambda_{1}$ is reduced to $1000 \mu \mathrm{m}$ by a branching mechanism. During the next step $(G=$ $6.24 \mathrm{~K} \mathrm{~mm}^{-1}$ ), $\lambda_{1}$ has reduced to $429 \mu \mathrm{m}$ (see Fig. 9(d)). At the highest $G, 18 \mathrm{~K} \mathrm{~mm}^{-1}$, the 2 initial primaries have branched into 10 primaries, with a $\lambda_{1}$ of $300 \mu \mathrm{m}$, or one fifth of the initial spacing (see Fig. 9(e)).

As can be seen in Fig 8 (b), a wide variation in primary spacing is predicted over the thermal gradient range of 1 to $18 \mathrm{~K} \mathrm{~mm}^{-1}$. However, the spacing changes in steps rather than smoothly and the upper band is hugged throughout this process. The average increase in $G$ before the spacing responds is $1.5 x$ the prior value.

The thermal gradient was then ramped down from $18 \mathrm{~K} \mathrm{~mm}^{-1}$ (point 'e') back to its initial value $1 \mathrm{~K} \mathrm{~mm}^{-1}$. As shown in Fig. $8 \mathrm{~b}$, the variation of $\lambda_{1}$ first undergoes a hysteresis period without any change. Elimination of existing dendrites does not start until $G$ decreases to $1.62 \mathrm{~K} \mathrm{~mm}^{-1}$ when the spacing is changed by an overgrowth mechanism (see Fig 9h). Decreasing $G$ further results in more dendrites being overgrown, leading to an increase in spacing. At point 'h', $G$ is restored to its original value, but the final value of $\lambda_{1}$ is $500 \mu \mathrm{m}$, one third of the initial value.
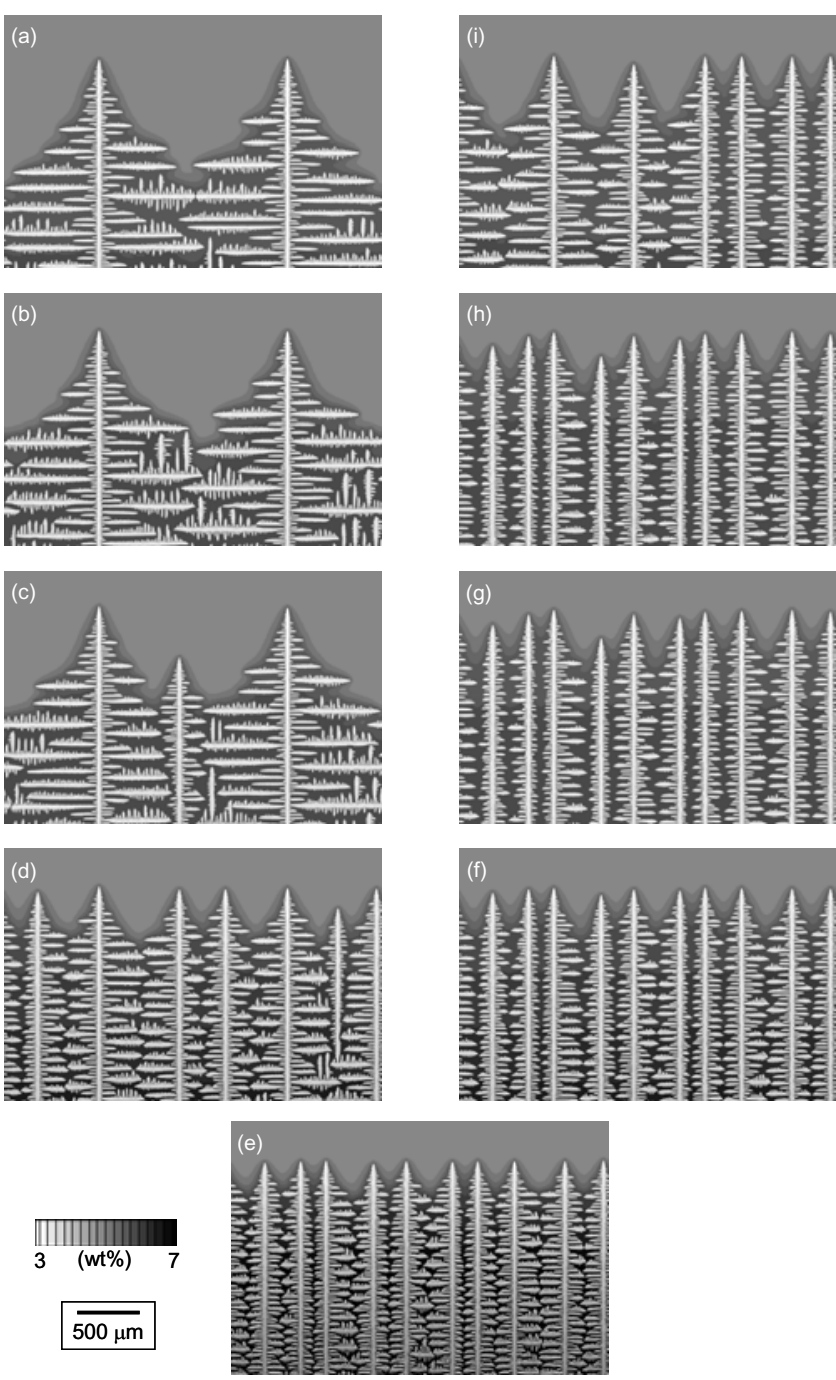

Fig. 9 Predicted dendritic structures corresponding to the thermal history given in Fig. 8, with a thermal gradient of: (a) 1.00 , (b) 1.96 , (c) 2.16 , (d) 6.24 , (e) 18.00 , (f) 6.24, (g) 2.16 , (h) 1.96 , and (i) $1.00 \mathrm{~K} \mathrm{~mm}^{-1}$.

In summary, when the thermal gradient increases, the primary spacing follows the upper bound of the allowable spacing range; when the gradient decreases the spacing hugs the lower bound. A hysteresis in spacing was observed. This conclusion may be industrially important; if either a fine or coarse spacing is desired, a tailored spacing could theoretically be produced by approaching steady state growth conditions via a different thermal history. For example, if the finest spacing possible for a given set of steady state growth conditions was desired to minimize solutionizing times, these thermal conditions could be approached from an initially higher gradient. The spacing predicted by these $2 \mathrm{D}$ results would then be one third that obtained by approaching from a lower gradient, reducing the solutionizing time by almost an order of magnitude. (It should be noted that prior steady state simulations in $3 \mathrm{D}$ by the current authors suggest the factor is closer to two rather than three between the upper and lower bound [19].)

Note that when decreasing $G$, there is an initial large lag, after which the spacing changes after fewer step changes in gradient 
than when increasing $G$. Overgrowth appears to be a faster and more sensitive mechanism then branching. This can be explained by the requirement of branching to form a tertiary arm, which can outgrow the secondary arms, while overgrowth occurs near the tips of the primaries. In practice, due to the difficulty in branching, new grains may nucleate in the advancing dendritic network, leading to the formation of stray grains. This will be examined in detail in the next section.

\section{Influence of Primary Spacing on Stray Grain Formation}

Although the finest possible primary spacing may be desirable from the perspective of minimizing solutionizing times, one of the other possible implications of altering the structure could be a change in the propensity to form stray grains. To study this an additional series of simulations was performed with heterogeneous nucleation included and for different initial spacings, ranging from 100 to $600 \mu \mathrm{m}$. Heterogeneous nucleation was included using a Gaussian nucleation distribution to relate the grain density with the local solute adjusted undercooling [12]. A mean nucleation undercooling of $12 \mathrm{~K}$ with a standard deviation of $1 \mathrm{~K}$ was assumed, with a maximum nucleation density of $6.0 \times 10^{10} \mathrm{~m}^{-3}$. A constant thermal gradient of $12 \mathrm{~K} \mathrm{~mm}^{-1}$ was used, while the pulling velocity was increased from the lower limit to the upper limit of the stable range predicted by Wang et al [19].

The expected result based on the conclusions of prior studies was for the propensity for stray grains to be greater at smaller spacings. The reason for this expected behavior was that as spacing decreases the solute fields interact more, increasing the tip undercooling [10] and hence the size of the region in which heterogeneous nucleation can occur. Although the model predicts this increasing undercooling ahead of the tips, the simulations actually predict that the propensity for stray grains decreases. The reason for this is shown in Fig. 10. This figure illustrates that the largest undercooled region is located in the dendrite groove behind the tips, not ahead of the columnar tip as assumed in theoretical studies. A detailed analysis of the compositionally corrected undercooling along the lines AA and BB in Fig 10 is shown in Fig. 11. The analysis shows that the maximum undercooling along the line $\mathrm{AA}$ is $9.2 \mathrm{~K}$ ahead of the tip, while the maximum undercooling along the line $\mathrm{BB}$ is $11.3 \mathrm{~K}$ in the dendrite groove. The undercooling difference between the columnar tip and maximum undercooled region is $2.1 \mathrm{~K}$ for a primary spacing of $600 \mu \mathrm{m}$. As shown in Fig. 10, this difference is dependent on the depth of the dendritic groove. Dendrites with larger spacing have deeper grooves between them; therefore, under the same thermal conditions, larger spaced dendrites are more prone to form new grains. The new grains usually do not have the same orientation as the columnar dendrites, leading to the formation of undesirable high angle grain boundaries. To avoid the formation of this defect, seeds with the smallest allowable spacing are recommended. If a small spacing is obtained, the extent of both microsegregation and stray grain formation will be reduced. This can also profoundly affect the homogenization heat treatment and improve the mechanical properties of the solidified products.

\section{Conclusions}

History dependent primary dendrite spacing during directional solidification was investigated using a combined cellular automaton-finite difference model. This model simulates the key

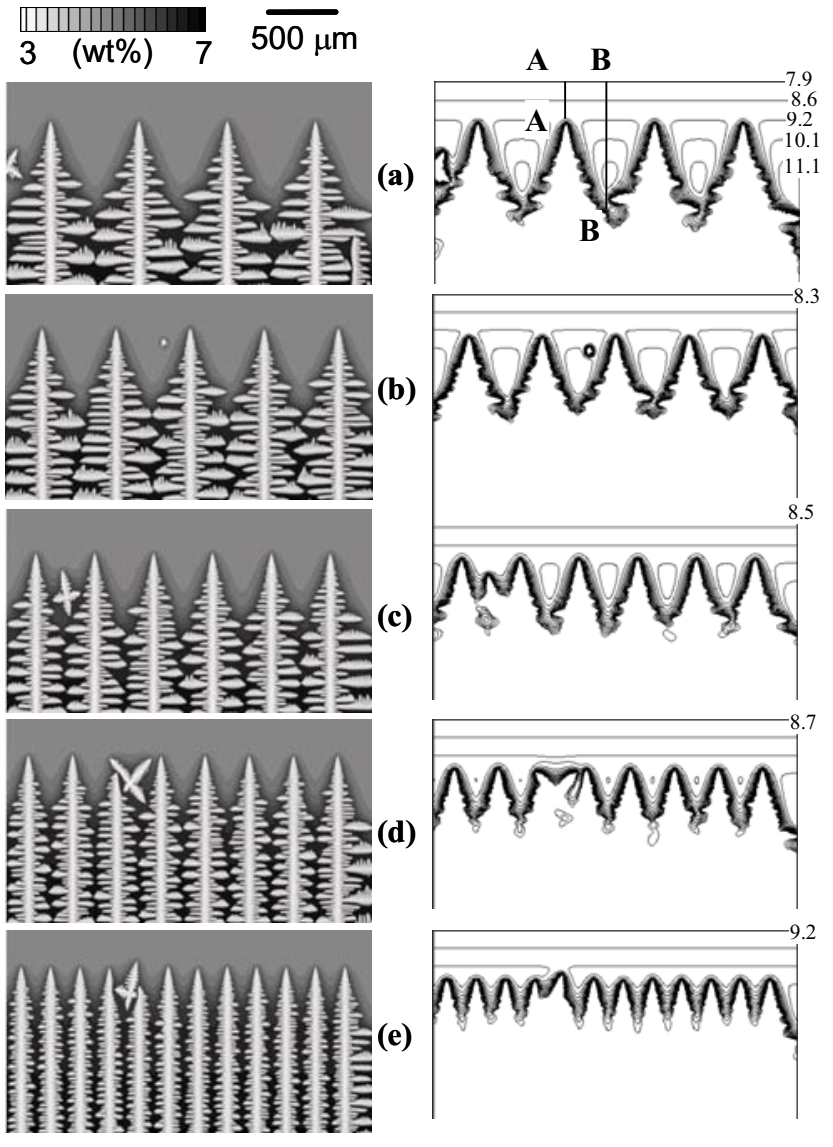

Fig. 10 Predicted dendritic fronts (left hand column) and the correspondent local undercooling contour plot (right hand column, counter spaced about $1 \mathrm{~K}$ apart) for different initial spacings at velocities of (a) 11 (b) 28 (c) 80 (d) 130 and (e) $1000 \mu \mathrm{m} \mathrm{s}^{-1}$

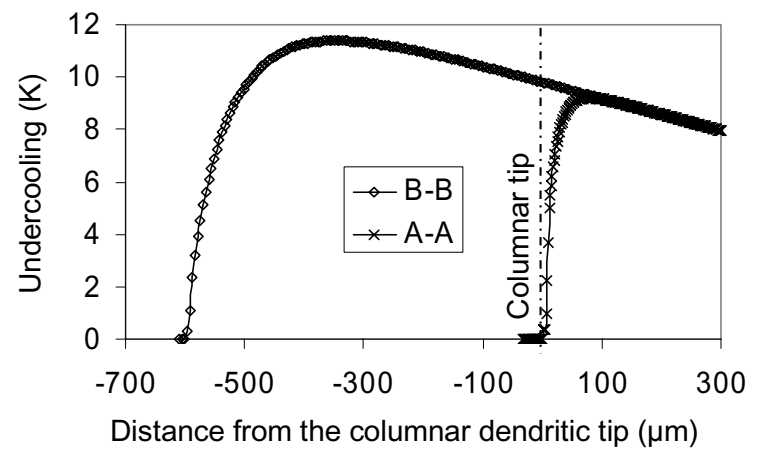

Fig 11 Undercooling in a dendrite groove along the lines A-A and B-B as indicated in Fig 10(a).

phenomena occurring during dendritic growth, including overgrowth and branching.

The dependence of primary spacing during columnar dendritic growth upon the thermal history was simulated for thermal gradients ramped from 1 to $18 \mathrm{~K} \mathrm{~mm}^{-1}$. The pulling velocity was kept constant. Changes in primary spacing through both branching and overgrowth mechanisms were quantified. It was found that a range of stable spacings were possible for any given growth 
condition, with the maximum spacing about three times the minimum spacing. When monotonically increasing the thermal gradient, the primary spacing always stays at the upper bound of this range. When monotonically decreasing the gradient the lower bound is followed.

The dependence of the heterogeneous nucleation of equiaxed grains upon primary arm spacing was also studied to investigate the propensity for forming high angle grain boundaries (HABs). It was found that within the range of stable spacings, HABs are most likely to form if the spacing is at the upper bound. Therefore, to minimize both homogenization times and to prevent HABs, thermal histories should be chosen where the steady state growth condition in a furnace is approached from a higher gradient state.

\section{Acknowledgements}

The authors would like to thank the EPSRC (GR/R78992 and GR/N14132), Rolls-Royce plc and Cannon-Muskegon, for financial support. They also gratefully acknowledge the assistance of their colleagues in the Materials Processing Group, Imperial College London.

\section{References}

1. M. McLean, Directionally Solidified Materials for High Temperature Service. (London, The Metals Society, 1983), 21-34.

2. W. Kurz and D. J. Fisher, Fundamentals of Solidification, Third ed. (Aedermannsdorf, Trans Tech Pub., 1992), 85-88.

3. C.E. Campbell, W.J. Boettinger, and U. R. Kattner, "Development of a diffusion mobility database for Ni-base superalloys", Acta Mater., 50, (2002), 775.

4. J.D. Hunt, "Keynote Address: Cellular and primary dendrite spacings" (Paper presented at Int. Conf. Solidification, Met. Society, London, 1979), 3-9.

5. K. Somboonsuk and R. Trivedi, "Dynamic Studies of Dendritic Growth", Acta Mater., 33, (1985), 1051.

6. W. Huang, X. Geng, and Y. Zhou, "Primary Spacing Selection of Constrained Dendritic Growth", J. Cryst. Growth, 134, (1993), 105.

7. D. Ma, "Modeling of Primary Spacing Selection in Dendrite Arrays during Directional Solidification", Metall. Mater. Trans. B, 33, (2002), 223.

8. J.A. Warren and J.S. Langer, "Stability of Dendritic Arrays", Physical Review A, 42, (1990), 3518.

9. J.A. Warren and J.S. Langer, "Prediction of Dendritic Spacings in a Directional- Solidification Experiment", Phys. Rev. E, 47, (1993), 2702.

10. J.D. Hunt and S.Z. Lu, "Numerical Modeling of Cellular/Dendritic Array Growth: Spacing and Structure Predictions", Metall. Mater. Trans. A, 27, (1996), 611.
11. W.J. Boettinger and J.A. Warren, "The Phase-Field Method: Simulation of Alloy Dendritic Solidification during Recalescence", Metall. Mater. Trans. A, 27, (1996), 657.

12. C.-A. Gandin, M. Rappaz, and R. Tintillier, "ThreeDimensional Probabilistic Simulation Of Solidification Grain Structures: Application To Superalloy Precision Castings", Metall. Trans. A, 24, (1993), 467.

13. W. Kurz, B. Giovanola, and R. Trivedi, "Theory of Microstructural Development during Rapid Solidification", Acta Metall., 34, (1986), 823.

14. P.D. Lee, "Simulation of Defect Formation During the Solidification of Aluminium Shape Castings", (Paper presented at Oxford-Kobe Materials Seminar, Solidification and Casting, Oxford, 1999), 117.

15. P.D. Lee, D. See, and R.C. Atwood, "Porosity Formation During Solidification - A Comparison of Micro modelling Approaches" (Paper presented at the conference of Cutting Edge of Computer Simulation of Solidification and Casting, Osaka, Japan, ISIJ, 1999), 97.

16. W. Wang, et al. "An Investigation of the Effect of Perturbations on Dendritic Growth in Nickel Base Superalloys" (Paper presented at the International Symposium on Liquid Metal Processing and Casting, Santa Fe, New Mexico, SF2M, 2001), 267.

17. W. Wang, et al. "A Microstructural Model of Competitive Growth in Nickel Based Superalloys", Int. J. Cast Met. Res., 15, (2002), 269.

18. W. Wang, et al. "Simulation of Dendritic Growth in the Platform Region of Single Crystal Superalloy Turbine Blades", $J$. Mater. Sci., 38, (2003), 4385.

19. W. Wang, P.D. Lee, and M. McLean, "A Model Of Solidification Microstructures In Nickel Based Superalloys: Predicting Primary Dendrite Spacing Selection", Acta Mater., 51, (2003), 2971.

20. C.-A. Gandin and M. Rappaz, "A 3D Cellular Automaton Algorithm for the Prediction of Dendritic Grain Growth", Acta Mater., 45, (1997) 2187.

21. L. Beltran-Sanchez and D.M. Stefanescu, "Growth of solutal dendrites: A cellular automaton model and its quantitative capabilities", Metal. Mater. Trans. A, 34, (2003),367.

22. W. Wang, "A Mathematical Model of Dendritic Microstructures in Nickel-Based Superalloys", (Ph.D. Thesis, Imperial College London, 2003), 59-69.

23. W. Kurz and D.J. Fisher, "Dendrite Growth at the Limit of Stability: Tip Radius and Spacing", Acta Mater., 29, (1981), 11. 
